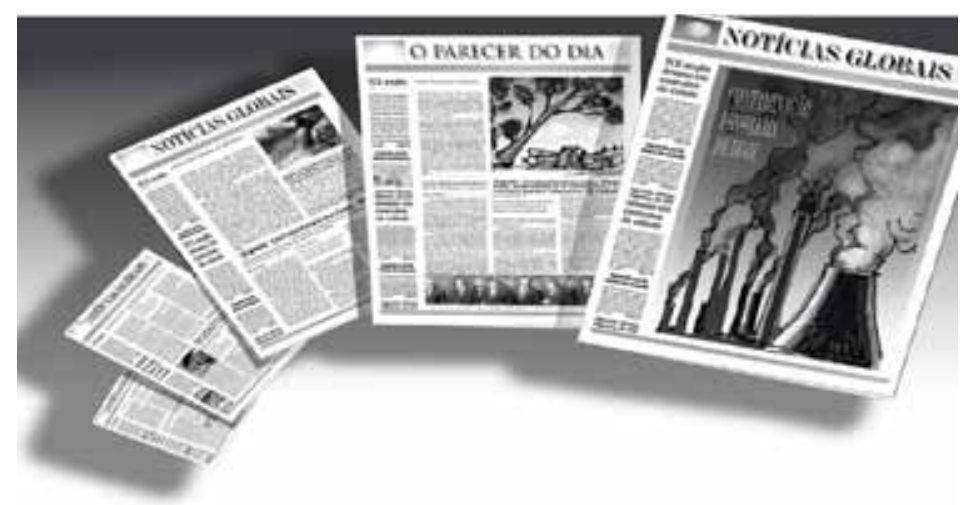

\title{
Las políticas ambientales en la prensa brasileña
}

\section{Environmental Public Policies in the Brazilian Press}

\begin{abstract}
EMERSON URIZZI CERVI. Departamento de Comunicación y Periodismo, Universidad Estatal de Ponta Grossa, Brasil. (ecervi7@ggmail.com) MICHELE GOULART MASSUCHIN. Universidad Federal de Paraná, Brasil. (mimassuchinahotmail.com)
\end{abstract}

- Recibido: 23/08/2011. Aceptado: 28/11/2011

\section{RESUMEN}

Este artículo pretende identificar cómo son abordadas las políticas públicas ambientales en la prensa brasileña desde 2000 hasta 2009, período en que se formuló y ejecutó el "Segundo Plano Nacional para o Meio Ambiente" (Pnma II). Se analizan las noticias de dos medios con diferentes áreas de circulación: Folha de São Paulo, producido en São Paulo y de circulación nacional y Gazeta do Povo, de Curitiba y con circulación en el estado de Paraná. La metodología utilizada es el análisis cuantitativo de contenido para medir la distribución de los textos en las categorías de las variables analizadas. La hipótesis inicial es que la cobertura periodística aparece centrada en ciertos temas y que las etapas del ciclo de políticas y el tratamiento es diferente en los dos periódicos.

Palabras clave: Política pública, medioambiente, medios impresos, ciclo de la política.

\section{ABSTRACT}

This article aims to identify how environmental public policies are addressed in the Brazilian press from 2000 to 2009, years during which was formulated and implemented the "Segundo Plano Nacional para o Meio Ambiente" (Pnma II). Two newspapers with different circulation were observed: Folha de São Paulo produced in São Paulo and of national scope and the Gazeta do Povo, of Curitiba and with circulation in the state of Parana. The methodology used is quantitative content analysis, which can measure the distribution of the texts in the categories of the variables analyzed. The initial hypothesis is that the coverage is centered on certain issues and the stages of the policies cycle, and that the treatment is different in the two newspapers.

Keywords: public policy, environment, newspapers, policy cycles.

Esta investigación cuenta con apoyo financiero del Programa REUNI del gobierno federal de Brasil y es parte de los trabajos producidos por las universidades Federal del Paraná y la Estadual de Ponta Grossa. 


\section{INTRODUCCIÓN}

Este trabajo analiza cómo las políticas públicas ambientales han ganado espacio en los debates públicos, específicamente en el debate promovido por los medios de comunicación durante el período de 2000 a 2009, cuando se implementó y ejecutó el "Segundo Plano Nacional para o Meio Ambiente" (Pnma II). La investigación apunta los temas y las etapas del ciclo político que ganaron espacio en los debates de las políticas ambientales en los 10 años estudiados. Observando las acciones implementadas durante la última década, se hace evidente que el tema ha adquirido importancia en el debate público, sea por el estancamiento en la aplicación de nuevas leyes o por medidas eficaces, entre otras cuestiones.

En paralelo con los asuntos ambientales, las acciones del gobierno se han convertido en una cuestión pública, como parte de la agenda de discusión de los medios de comunicación, de la sociedad y de los políticos. La visibilidad de estos en los medios genera la idea de que ganan importancia y luego influyen en la mejora del debate público mediante la difusión de la información. Estas ideas parten del presupuesto de que el debate público tiene como base el proceso de transferencia de agenda de los medios hacia la agenda pública (McCombs, 2004). Aunque no influyan directamente en la formación de la opinión pública, es la principal fuente de información de la sociedad actual y tiene el poder de presentar u ocultar ciertos temas (McCombs y Shaw, 1972).

Los medios de comunicación son considerados como un espacio donde se forma el debate público y donde se puede ver cuáles son los temas más importantes y la cantidad de espacio que ocupan en las discusiones políticas. Y, además, tienen el papel fundamental en informar a los ciudadanos, ya que estos utilizan la información recibida como base para orientar sus comentarios al respecto de asuntos de interés público.

\section{METODOLOGÍA}

La investigación utiliza métodos cuantitativos con el objetivo de identificar cómo las políticas ambientales están ganando espacio y hacen parte de la agenda temática de los medios brasileños. La elección del método se relaciona con el objetivo de la investigación, que consiste en relacionar los hechos de la realidad con los conceptos y las teorías existentes. Se hacen necesarias varias directrices, como la exigencia de un gran número de casos para poder detectar diferencias y para hacer inferencia en la realidad (Davis, 1976). Cabe señalar también que la técnica de recolección es el análisis de contenido de los periódicos.
Se trabajó con una muestra por semana compuesta, donde fueron analizadas 520 ediciones de cada periódico y recogidas 813 entradas, siendo 446 de la Folha de São Paulo y 367 en la Gazeta do Povo.

De la investigación analiza la cobertura periodística de los medios impresos con un conjunto de variables divididas en categorías. La cuestión central -que será discutida en este artículo- está relacionada con el tema de las políticas públicas y los pasos del ciclo político. Ellos permiten identificar cómo son las discusiones acerca de las políticas ambientales, cuáles son los temas prioritarios en el debate (sostenibilidad, educación ambiental, legislación, devastación, etc.) y las fases del ciclo político (preparación, formulación, implementación, ejecución y evaluación) que se son destacados en los periódicos brasileños.

Los datos totalizan una muestra de 1.040 ediciones de los dos periódicos, con catalogación solo de aquellos textos que hacen referencia a las acciones gubernamentales para el medioambiente. La investigación resultó en un total de 813 textos, siendo 446 del el periódico Folha de São Paulo y 367 de la Gazeta do Povo. Como el espacio de tiempo es muy amplio, la investigación se hace por muestra, que según Bauer (2002) es una muestra por semana compuesta (una edición semanal de todos los periódicos en secuencia continua). El período de análisis -como ya se indicó-incluye los años de 2000 a 2009, lo que se justifica por la creación del segundo "Plano Nacional de Meio Ambiente", que incluye medidas de los niveles federales, estatales y locales que fueron practicadas en todo el país.

La investigación cuantitativa ofrece la posibilidad de correlacionar las variables y hacer un análisis multivariado, basado en la información recogida en los textos a través de la utilización de las estadísticas que, según Davis (1976), justifica el tamaño de la muestra utilizada. En el análisis cuantitativo se puede buscar en un gran número de individuos (en este caso, los textos periodísticos), la observación de muchas de sus características, ya que este método reduce una gran cantidad de información en los indicadores, cuantificando y haciendo su interpretación.

En este texto se hace el análisis de dos variables específicas: los temas de las políticas y los pasos del ciclo político, que permiten identificar en qué parte del proceso político las acciones del gobierno para el medioambiente están presentes en los medios de comunicación. Estas variables se relacionan con otras: el espacio que los textos ocupan, la posición en la página, la presencia de imágenes y la cantidad de entradas respecto de cada tema. 


\section{LOS MEDIOS DE COMUNICACIÓN EN EL DEBATE ACERCA DE LAS ACCIONES DEL GOBIERNO}

En la sociedad que se establece desde principios del siglo $\mathrm{XX}$, los medios de comunicación empezaron a desempeñar un papel central en la difusión de información, principalmente con la ampliación de la audiencia de los periódicos y con la popularización de la radio y la televisión. De acuerdo con Miguel (2002), la relación entre la política y los medios de comunicación se ha intensificado en los últimos años y estos cada vez ocupan un espacio más prominente en la sociedad. Han adquirido un importante papel de mediador del debate en la esfera pública, es decir, actúan como enlace entre la esfera política y la sociedad civil. La visibilidad que se da en los medios de comunicación a algunas cuestiones contribuye a un diálogo público generalizado (Maia, 2002). Este proceso de transferencia de la agenda de la prensa a la agenda pública se llama agenda-setting, un término ya utilizado en la década de 1970 por McCombs y Shaw (1972).

Por otra parte, la comprensión de los medios de comunicación como un espacio para el debate y actor influyente en los cambios que se producen en la esfera pública, los presenta como un factor importante en las sociedades, desde su papel como mediador, lo que potencializa el debate público de los temas y la confrontación de ideas. Sin embargo, debe tenerse en cuenta que, al retratar los hechos y acontecimientos, los medios no los representan con exactitud en sus características reales debido al proceso de producción de las noticias, a elementos tales como el tipo de fuentes, el mercado, la sociedad, la cultura, etc. (Shoemaker \& Voz, 2009), que son responsables por la construcción de la agenda de los medios (McCombs, 2004). De acuerdo con Habermas (2006), estos seleccionan contenidos políticamente relevantes que tienen influencia en la formación de la opinión pública y en la distribución de interés. A través de esta selección de temas, son capaces de cristalizar solo una parte del debate y, por lo tanto, actuar como constructores de una realidad específica.

Sin embargo, McCombs (2004) subraya que el proceso de agendamento se produce con mayor eficacia cuando los individuos buscan información para orientar sus opiniones, por ejemplo, para saber más acerca del tema medioambiental. Dado que esta búsqueda de orientación está vinculada con el tema pertinente para el individuo y la incertitumbre al respecto, estos conceptos están relacionados con el ambiente informacional de los ciudadanos. Y cuanto más utilizan las noticias para averiguar acerca de ciertas cuestiones, más están propensos a internalizarlas, pero lo que aquellos que no tienen ningún interés en este asunto no sufren influencia significativa.

En este estudio abordamos un problema de interés público, es decir, qué es relevante para la comunidad. Las acciones del gobierno en materia ambiental son importantes si llegan a la población, incluso tratándose de una ley ambiental o un proyecto para materiales reciclables. Por lo tanto, la necesidad de información acerca del tema afecta a los ciudadanos en busca de orientación. Según McCombs (2004), los temas más lejanos a la persona, que no se discuten en el círculo de amigos y familiares, tienen una correlación más estrecha entre las agendas de los medios de comunicación y el público, debido a necesidad de adquirir información. En el caso de las políticas ambientales, que es un tema que importa a las personas, pero que no tienen información, hay necesidad de orientación por parte de los medios de comunicación. Para Aruguete (2005), los eventos que están fuera del alcance de los individuos llegan a ellos a través de la prensa. En la medida que los sistemas de comunicación transmiten noticias de temas de interés público, tales como las acciones gobernamentales para el medioambiente, contribuyen con el debate en la sociedad y con el establecimiento de la agenda de temas públicos a través de su capacidad de programar.

Aunque los medios no son la única forma de mediación de los asuntos públicos con la sociedad, superan a los políticos, investigadores, ONGs y movimientos sociales, que pertenecen a esferas específicas. De acuerdo con Habermas (2006), el poder de los medios de comunicación se basa en tecnologías de la información de masas y a así llegan a un gran número de personas. Una noticia acerca de la aplicación del derecho ambiental, por ejemplo, llega a un público mayor que una campaña hecha por un movimiento social.

Habermas además otorga a los medios el poder de ser líderes de opinión. Según el autor, el vehículo selecciona y ofrece contenido político relevante y participa de la conformación de la opinión pública. Como Aruguete (2005), Habermas expone las formas en que los medios de comunicación tienen el poder: la elección de la información y el formato, la forma y el estilo de los programas y los efectos de la difusión de la información.

El espacio ocupado por el tema, la posición en las páginas, la presencia de gráficos y el encuadre son importantes en la construcción del debate público. McCombs (2004) considera que el porcentaje de espacio ocupado por el tema en una edición ayuda a explicar el proceso de agendamento, ya que el mayor espacio llama más la atención del lector. Además, el 
autor señala la diferencia de la ubicación: las noticias que están en la primera página se leen dos veces más que las que aparecen en las internas y lo mismo ocurre con la posición en los cuadrantes. Otro factor que también tiene un impacto en el agendamendo es la presencia de la ilustración gráfica, ya que tiene un efecto atractivo para el lector.

Segun Aruguete (2005), no es para todos los temas que los medios de comunicación tienen el poder de programar el debate público, sino sólo aquellos que reciban más importancia. Las temáticas (sostenibilidad, conservación, educación, ambiental, etc) tienen la posibilidad de obtener diferentes características a partir del formato en que aparecen en el periódico, generando un impacto diferente en el lector. Esto está relacionado con la visibilidad, ya que en la medida en que el tema es más relevante ocupa más espacio y puede producir un efecto mayor en la agenda pública. Esto justifica la investigación de la cobertura que los medios de comunicación hacen de los asuntos públicos.

\section{EL MEDIOAMBIENTE \\ COMO TEMA EN LOS PERIÓDICOS}

Las políticas ambientales empezaron a aparecer más destacado después de la ECO-92 en Brasil. En la medida en que la sociedad discute algunas cuestiones, los medios de comunicación tienden a seguir lo que está aconteciendo. Esto explica por qué el tema medioambiente ha ganado espacio en la prensa en los últimos años. Los periódicos hablan todos los días de la crisis ambiental y los problemas ecológicos (Abreu, 2006).

Algunos autores dicen que el periodismo ambiental tiene de poco más de 30 años. El interés por el tema surgió a partir de 1970, con el trabajo del movimiento ambiental en Brasil. "El periodismo ambiental nació junto con los movimientos ecologistas y los eventos patrocinados por las Naciones Unidas en las últimas décadas" (Souza, 2005, p. 33). Por otra parte, sólo después de la Conferencia de Estocolmo en 1972 fue posible una mayor cobertura. Según Abreu (2006), los temas discutidos hoy en día, como el calentamiento global, la deforestación, la contaminación del agua, la contaminación del aire, exceso de residuos y las energías renovables, sólo se convirtieron en parte del periodismo ambiental después esa Conferencia.

Para Bonfiglioli (2006), los medios de comunicación fueron muy imporantes para hacer del tema ambiental un asunto de interés público. Durante las décadas del 70 y 80 hubo otras reuniones que trataron el tema de medioambiente y la influencia de la prensa, pero para la periodista Eliana de Souza Lima (2002) fue solamente después de 1992 que el periodismo ambiental surgió, como resultado de la ECO-92, celebrada en Río de Janeiro. El evento hizo que la prensa produjera folletos y suplementos para responder a la demanda de noticias al respecto (Trigueiro, 2008). Sin embargo, Arruda (2006) considera a Randau Marques, del Jornal da Tarde, como el primero en destacar la cobertura del medioambiente en Brasil, ya en 1968. Por otra parte, en términos de los pioneros en el periodismo ambiental, se menciona también la revista Realidade de 1972. El estado de Rio Grande do Sul también debe ser considerado como un pionero en la lucha por los temas ambientales en Brasil (Alves, 2002), ya que los periódicos regionales son los principales responsables por la divulgación del tema y la lucha por la preservación del medioambiente en la década de 1970

Castro (2004) explica el desarrollo del periodismo ambiental desde la creciente preocupación pública. Sin embargo, Arruda (2006) defiende la infidelidad de los medios con el tema, acercándose a él solo cuando las tragedias ocurren. La crítica para los medios de comunicación es, en parte, debido al espacio que dan a noticias que solo se relacionan con un desastre o accidente ambiental (Aguiar, 2005). Y Villar (1997) señala que "la prensa brasileña raras veces se ocupa de los problemas ambientales con profundidad en la agenda de discusión pública. Las excepciones son resultado del esfuerzo personal de pocos periodistas" (p. 1).

Según Villar (2004), lo que sigue dominando en los noticieros sobre los problemas urbanos es la cobertura puntual, lo que aumenta en tiempos de crisis (como fue el caso de las inundaciones en la costa de Santa Catarina en 2008, las inundaciones casi normales en las grandes ciudades, el tsunami en diciembre de 2004 , etc.) y el poco espacio para el debate, el análisis, la investigación de las causas, las interpretaciones y la presentación de soluciones a los problemas. Para Tautz (2005), el periodismo también tiene la función de mirar si la legislación ambiental y las políticas estan siendo puestas en práctica.

Debido a la amplitud del tema del medioambiente y la realización de la ECO-92 en Brasil, Villar (1997) considera el periodismo ambiental como algo irreversible en los medios por una cuestión de marketing, aunque todavía es necesario mejorar el contenido. En Brasil, en la última década del siglo XX, la cobertura sobre el tema pasó por tres etapas distintas según Tosi y Villar (2001). Los autores caracterizan a los años 90 a 92 como el auge de la discusión. Un momento más tarde, la califican de "resaca", debido a la reducción de las noticias sobre el tema. En los últimos años del siglo, el 
asunto pasó a componer las directrices debido a la aparición de grandes corporaciones que contaminan y el creciente interés en el Sello Verde.

También hay otra clasificación. Para explicar la trayectoria de la producción en esta área del periodismo, Aguiar (2005) define cuatro formas de representar el medioambiente. En los años 60, fue representado como una crisis de participación; en la década siguiente, como una crisis de supervivencia; en los años 80 , como una crisis cultural; en la última década del siglo lo que marca son las crisis (calentamiento global, la deforestación, la contaminación del agua, la contaminación del aire, el exceso de residuos).

La aparición de nuevos canales de información, como periódicos en Internet, las radios comunitarias y locales, por ejemplo, proporcionan un espacio para una multiplicidad de voces, dando lugar a movimientos ambientalistas, ONGs, etc. (Bueno, 2004). Según Alves (2002, p. 8), es necesario que los medios de comunicación hagan periodismo ambiental relacionado con "la ecología, el medioambiente y nuestras experiencias cotidianas" para que las personas tengan más información. Teresa Urban, periodista y ambientalista, habla de los problemas causados cuando las personas carecen de información en la materia. Según ella, la ignorancia convierte el ciudadano en depredador; y la ignorancia de las causas y consecuencias dificulta el ejercicio de la ciudadanía.

\section{EL DESARROLLO DE POLÍTICAS PARA EL MEDIOAMBIENTE}

Para un tema como el medioambiente, ganar la atención del gobierno y componer la agenda política debe ser considerado importante para mucha gente. Segun Capella (2006), un tema se convierte en parte de la agenda del gobierno cuando despierta la atención y el interés también de los políticos. En el caso del tema ambiental, podemos considerar que a finales del siglo XX, con los descubrimientos científicos y la investigación, el público empezó a prestar más atención a la cuestión del medioambiente. Las políticas necesitan representar valores comunes y tener una aceptación del público en general. En el caso del medioambiente, las políticas creadas en este sector deben tener equilibrio, pues hay problemas como las diferencias entre el Ministerio de Medio Ambiente y Agricultura, por ejemplo.

Según Souza (2006), la política pública, después de formulada, es dividida en planes, programas y proyectos; cuando se implementa (se pone en acción), está sujeta a siste- mas de acompañamiento y evaluación. Para ilustrar, se puede utilizar la creación del "Plano Nacional de Meio Ambiente", surgido en los años 90, que incluye una variedad de acciones, actividades y proyectos en los tres niveles de gobierno, que tuvieron diez años de duración.

Pero, pese a la infinidad de cuestiones y problemas relacionados con el medioambiente, el tema no es considerado un foco en las políticas públicas (Lima, 2008), aún sabiendo que debe ocupar un lugar destacado tanto en la sociedad como en la política, ya que interfiere en otras acciones de la administración pública. Para el autor, esta falta de atención a la política ambiental es el resultado de un proceso histórico y cultural. Pero en las últimas décadas, un reflejo de la Eco-92, el gobierno federal, en colaboración con otros sectores políticos, creó nuevas leyes, programas y metas para el país. En este contexto, nació el "Plano Nacional de Meio Ambiente", que empezó en 2000 y finalizó en 2009.

El programa fue instituido con el objetivo de fortalecer el medioambiente nacional y sus organismos de ejecución, tales como el IBAMA, las secretarias del medioambiente, las agencias estatales y organizaciones del medioambiente. La segunda etapa del trabajo centró los esfuerzos en la capacidad de gestión de los Estados y el Sistema Estatal de Medio Ambiente. El Pnma II fue financiado a través de un convenio entre el Gobierno del Brasil y el Banco Mundial, que resultó en 300 millones de dólares para proyectos de implantación y desarrollo de las acciones en todo el país.

Observando las actividades gubernamentales realizadas durante la última década, es evidente que el tema ha adquirido importancia en el debate público, aunque todavía haya un punto muerto en la aplicación de nuevas leyes y en las medidas eficaces, entre otras cuestiones. Sin embargo, las acciones del gobierno se han convertido en una cuestión pública, como parte de la agenda de discusión de los medios de comunicación, la sociedad y los políticos.

\section{ALGUNOS RESULTADOS}

\subsection{VISIBILIDAD DEL TEMA EN LOS MEDIOS ANALIZADOS}

Como el estudio se refiere a dos periódicos de diferentes ámbitos, los datos se presentan por separado. Además de mostrar, en general, cómo las políticas reciben visibilidad, se puedens hacer algunas consideraciones acerca de las diferencias en la producción periodística de cada diario. La Tabla 1 muestra el número de textos en los periódicos, así como el espacio ocupado.

Los datos muestran una diferencia de 79 unidades en 
Tabla 1. Número de textos y espacio en los periódicos (promedio/día)

\begin{tabular}{|c|c|c|c|c|}
\hline \multirow{2}{*}{ Medidas } & \multicolumn{2}{|c|}{ Folha de São Paulo } & \multicolumn{2}{|l|}{ Gazeta do Povo } \\
\hline & Espacio cm2 & $\mathrm{N}^{\circ}$ Entradas & Espacio $\mathrm{cm} 2$ & $\mathrm{~N}^{\circ}$ Entradas \\
\hline Promedio & 282,99 & 1,76 & 310,1492 & 1,54 \\
\hline Mediana & 173,75 & 1,00 & 240,0000 & 1,00 \\
\hline Desviación Estándar & 266,57 & 1,10 & 286,73144 & 0,82 \\
\hline Total & $126.215,00$ & 446,00 & $113.824,75$ & 367,00 \\
\hline
\end{tabular}

Fuente: elaboración propia

el número de entradas: en el diario con circulación nacional había 446 entradas, mientras que en la cobertura del periódico regional, 367. El espacio ocupado sigue el misma norma: la FSP con $126,215 \mathrm{~cm} 2$ y Gazeta do Povo con $113,824 \mathrm{~cm} 2$. Sin embargo, la mediana (que no es sensible a los números extremos) y el tamaño promedio de las entradas son más altos en la Gazeta do Povo (240 y 310 $\mathrm{cm} 2$ respectivamente). Esto significa que los textos en la FSP, aunque aparecen en mayor cantidad, tienden a tener menor tamaño, a ser más fragmentados. Una hipótesis es que se trata de un periódico nacional y por esto hay más temas que disputan espacio para aparecer con más importancia y así las politicas ambientales no son consideradas como tema tan pertinente cuando es relacionado con otros como educación, salud, campaña electoral, etc.

Otra hipótesis que puede explicar el mayor espacio ocupado por los textos en Gazeta do Povo es de naturaleza institucional, es decir, está relacionada con las caracteristicas de producción hecha por el medio. En la FSP no hay un espacio para la discusión ambiental, por lo tanto el tema parece dividido en varias secciones, desde política hasta policía, en casos de asuntos relacionados con la vida silvestre o la devastación. En cambio, la Gazeta do Povo tiene, los miércoles, una página de medioambiente. Por lo tanto, cuando la fecha de la muestra fue en miércoles, hay más texto que de costumbre y también tienden a tener mayor tamaño. Esto muestra que existe una demanda por este problema, es decir, también es más probable encontrar textos acerca del tema en otros días de la semana. Es importante tener en cuenta que el tamaño total del los periódicos -el número de páginas- es muy similar, por lo tanto es posible comparar en términos absolutos el espacio ocupado por el tema.

Pero la visibilidad está relacionada con otros factores, como la posición en la página, la presencia de imágenes y el formato en que los textos aparecen en el periódico. Estos factores influyen en el proceso de agendamento del asunto en el debate público. La Tabla 2 presenta un indicador denominado índice de visibilidad que tiene en cuenta cuatro variables: posición en la página, tamaño del texto, presencia de imágenes y formato de la entrada. Según Weaver, McCombs y Spellman (1975), es la valoración de los temas en los periódicos, a través del tamaño del título y su localización (primera página o las interiores) la que define el impacto en el debate. Se parte del supuesto de que los textos acompañados de imágenes tienen más visibilidad que los otros; que las entradas de la primera página tienen mayor visibilidad que las notas (pequeñas noticias), las que a su vez serán más visibles que las columnas y otras entradas en páginas interiores. Por otra parte, si el texto está en la primera mitad de la página (parte superior), tiene mayor visibilidad que en la segunda parte (mitad inferior). La Tabla 2 compara la visibilidad de las políticas públicas en los dos periódicos analizados.

Los datos refuerzan las conclusiones anteriores: que la Gazeta do Povo da más visibilidad a las políticas públicas ambientales en relación a la FSP. Comparando los dos diarios, el conjunto de las 
Tabla 2. Índice de visibilidad ${ }^{1}$ de los textos de política ambiental

\begin{tabular}{|c|c|c|c|c|}
\hline & \multicolumn{2}{|l|}{ Folha de São Paulo } & \multicolumn{2}{|l|}{ Gazeta do Povo } \\
\hline & Frecuencia & $\%$ & Frecuencia & $\%$ \\
\hline Muy baja & 139 & 31,2 & 81 & 22,1 \\
\hline Baja & 95 & 21,3 & 74 & 20,2 \\
\hline Promedio & 58 & 13,0 & 69 & 18,8 \\
\hline Alta & 121 & 27,1 & 100 & 27,2 \\
\hline Muy alta & 33 & 7,4 & 43 & 11,7 \\
\hline Total & 446 & 100,0 & 367 & 100,0 \\
\hline
\end{tabular}

Fuente: elaboración propia

variables utilizadas en este indicador muestra que las categorías de 'muy bajo' y 'bajo' en la FSP tienen porcentajes más altos. A su vez, ocurre lo contrario en las categorías 'medio', 'alto' y 'muy alto'. Esto significa que cuando las políticas públicas ambientales aparecen en la Gazeta tienen más visibilidad que cuando están en la FSP.

Para continuar, se presentan, en la Tabla 3, las frecuencias de los temas de políticas ambientales abordados por los periódicos. Se señala que las categorías de las variables fuera producidas con base en la literatura sobre políticas ambientales. Posteriormente, estos datos se relacionarán con el índice de visibilidad que se creó, con el objetivo de determinar si hay temas más visibles que otros. El cuadro muestra que, entre las categorias, hay algunos temas que destacan en la cobertura de los periódicos, como sostenibilidad y la preservación de los espacios naturales, que juntos represen$\tan 70,2 \%$ de las entradas en la FSP y $583 \%$ en la Gazeta do Povo.

En la FSP, sostenibilidad aparece con 32,1\% de las entradas y preservación, con 38,1\%. En la Gazeta, los mismos temas aparecen con $35,7 \%$ y $22,6 \%$. Tanto en uno como en otro diario hay una concentración de más del 50\% de las entradas en solo dos temas, señalando que la concentración de la FSP es mayor en más de 12 puntos porcentuales. Aunque la sostenibilidad tiene 35,7\% de las entradas de la Gazeta do Povo, el segundo tema aparece con 22,6\%. En FSP ambas cuestiones tienen porcentajes superiores al 30\%.

Estas informaciónes muestran que no hay equilibrio en la cobertura de los temas en los periódicos estudiados, lo que, en parte, contribuye a afirmar la hipótesis del estudio: que la cobertura de medioambiente se centra solo en algunos temas. Educación ambiental, por ejemplo, apareció muy poco y representa un porcentaje casi insignificante de los textos: en la FSP, un $0.4 \%$ y 2.2\% en la Gazeta do Povo. Enfatizamos que educación ambiental fue uno de los principales puntos del II Plano Nacional de Meio Ambiente, sin embargo no apareció en los periódicos. Esta concentración en ciertos temas en la agenda de los medios es el resultado de una serie de procesos internos y externos que influyen en la producción de noticias como ha señalado Shoemaker y Vos (2009) y McCombs (2004).

En el caso de la FSP, el aumento de la producción se explica por el crecimiento de las ciudades, especialmente de São Paulo y por su impacto en la preservación del medioambiente. Este tema fue abordado por el II Plan Nacional para el Medio Ambiente y, en consecuencia, después de desplegado, tuvo repercusiones en los medios de comunicación. Además, otra posible explicación es la producción de una noticia cada semana acerca de la calidad del agua de mar, que aparece en la cobertura desde el año 2001. Hay que tener en cuenta también que las acciones de IBAMA, el Ministerio de Medio Ambiente y otros organismos, tales como multas, confiscación de animales, entre otras, también se consideran como una acción en las políticas de preservación del medioambiente.

En el caso de la Gazeta do Povo, el entorno externo a los editores es un factor que contribuye para explicar la gran cantidad de
1 Antes de producir este índice, fue verificada la consistencia o confiabilidad del indicador, por medio del Coeficiente Alfa de Cronbach, que fue de 0,64 $064 \%$, lo que significa que el indicador mide el $64 \%$ de los posibles impactos. 
Tabla 3. Frecuencias de los temas de política ambiental en los periódicos

\begin{tabular}{|c|c|c|c|c|}
\hline & \multicolumn{2}{|c|}{ Folha de São Paulo } & \multicolumn{2}{|l|}{ Gazeta do Povo } \\
\hline & Frecuencia & $\%$ & Frecuencia & $\%$ \\
\hline Sostenibilidad & 143 & 32,1 & 131 & 35,7 \\
\hline $\begin{array}{l}\text { Preservación de Áreas } \\
\text { Ambientales }\end{array}$ & 170 & 38,1 & 83 & 22,6 \\
\hline Legislación & 50 & 11,2 & 69 & 18,8 \\
\hline Políticas/devastación & 64 & 14,3 & 52 & 14,2 \\
\hline Educación Ambiental & 2 & 0,4 & 8 & 2,2 \\
\hline Otros temas & 17 & 3,8 & 24 & 6,5 \\
\hline Total & 446 & 100,0 & 367 & 100,0 \\
\hline
\end{tabular}

Fuente: elaboración propia

noticias sobre las acciones sostenibles propuestas por el gobierno. Curitiba, donde el periódico es producido, es una ciudad conocida por su aspecto ecológico. En estos textos se destacan temas específicos y locales, tales como el reciclaje, el tratamiento de las aguas, la emisión de contaminantes, limpieza de las calles, entre otros que son recurrentes.

Por lo tanto, asumiendo que la agenda de los medios de comunicación se transfiere a la agenda pública, el debate que ocurre en la sociedad acerca de las acciones del gobierno para el medioambiente también tiende a concentrarse en los temas que ganan más énfasis en la cobertura. Para esto debe tenerse en cuenta la forma en que los textos de este tema aparecen en el periódico. Con este fin, la Tabla 4 muestra la relación de los temas que aparecen en los periódicos con el índice de visibilidad. Es posible percibir que los asuntos que más aparecen también son aquellos que ganan más espacio. Para cada periódico se realizó el análisis de forma independiente, mediante la prueba de Chi-cuadrado, que fue significativo sólo para el periódico Folha de Sao Paulo, con un nivel de significación de 0.000 y coeficiente muy alto $(36,647)$. En la Gazeta do Povo, la prueba mostró el resultado de 0.290, que está por encima del límite crítico (0.050), es decir, no existe una relación significativa entre las dos variables de la prueba. Por otra parte, el coeficiente también es menos que aceptable $(14,177)$. Esto significa que las cuestiones planteadas se distribuyen más equitativamente entre todas las visibilidades, es decir, no hay ningún tema que tienda a tener una relación más fuerte con cierta categoría de visibilidad. En cuanto al caso de la Folha de Sao Paulo, es necesario observar cuáles son los temas más relacionados con cierta visibilidad en detrimento de los demás. En este caso, cuando la relación es significativa, hay también residuos estandarizados que muestran qué categorías de las dos variables tienen una relación más estrecha.

Prestando más atención a los datos que corresponden a la FSP, y que apuntan a una relación significativa entre las variables, se observa que el tema áreas de preservación ambiental tiende a aparecer en textos de poca visibilidad, mientras que las políticas contra la devastación aparecen más en textos de alta visibilidad, en comparación con el porcentaje que se encuentran en otras categorías. Los temas sostenibilidad y legislación parecen más equilibrados entre todos los niveles de visibilidad. 
Tabla 4. Relación entre los temas y la visibilidad de los textos

\begin{tabular}{|c|c|c|c|c|c|c|}
\hline & & Sostenibilidad & Preservación & Legislación & $\begin{array}{l}\text { Política } \\
\text { devastación }\end{array}$ & Total \\
\hline \multirow{17}{*}{$\begin{array}{l}\text { Folha de São } \\
\text { Paulo }\end{array}$} & \multirow{3}{*}{ Muy baja } & 36 & 75 & 12 & 12 & 135 \\
\hline & & $25,2 \%$ & $44,1 \%$ & $24,0 \%$ & $18,8 \%$ & $31,6 \%$ \\
\hline & & $-1,4$ & 2,9 & $-1,0$ & $-1,8$ & \\
\hline & \multirow{3}{*}{ Baja } & 37 & 30 & 12 & 11 & 90 \\
\hline & & $25,9 \%$ & $17,6 \%$ & $24,0 \%$ & $17,2 \%$ & $21,1 \%$ \\
\hline & & 1,2 & $-1,0$ & .5 &,- 7 & \\
\hline & \multirow{3}{*}{ Promedia } & 20 & 14 & 11 & 8 & 53 \\
\hline & & $14,0 \%$ & $8,2 \%$ & $22,0 \%$ & $12,5 \%$ & $12,4 \%$ \\
\hline & & .5 & $-1,5$ & 1,9 & 0 & \\
\hline & \multirow{3}{*}{ Alta } & 36 & 41 & 11 & 30 & 118 \\
\hline & & $25,2 \%$ & $24,1 \%$ & $22,0 \%$ & $46,9 \%$ & $27,6 \%$ \\
\hline & &,- 6 & -.9 &,- 8 & 2,9 & \\
\hline & \multirow{3}{*}{ Muy alta } & 14 & 10 & 4 & 3 & 31 \\
\hline & & $9,8 \%$ & $5,9 \%$ & $8,0 \%$ & $4,7 \%$ & $7,3 \%$ \\
\hline & & 1,1 &,- 7 & .2 & -.8 & \\
\hline & \multirow{2}{*}{ Total } & 143 & 170 & 50 & 64 & 427 \\
\hline & & $100,0 \%$ & $100,0 \%$ & $100,0 \%$ & $100,0 \%$ & $100,0 \%$ \\
\hline \multirow{12}{*}{$\begin{array}{l}\text { Gazeta do } \\
\text { Povo }\end{array}$} & \multirow{2}{*}{ Muy baja } & 24 & 15 & 17 & 15 & 71 \\
\hline & & $18,3 \%$ & $18,1 \%$ & $24,6 \%$ & $28,8 \%$ & $21,2 \%$ \\
\hline & \multirow{2}{*}{ Baja } & 27 & 20 & 15 & 6 & 68 \\
\hline & & $20,6 \%$ & $24,1 \%$ & $21,7 \%$ & $11,5 \%$ & $20,3 \%$ \\
\hline & \multirow{2}{*}{ Promedia } & 27 & 10 & 17 & 9 & 63 \\
\hline & & $20,6 \%$ & $12,0 \%$ & $24,6 \%$ & $17,3 \%$ & $18,8 \%$ \\
\hline & \multirow{2}{*}{ Alta } & 33 & 29 & 14 & 16 & 92 \\
\hline & & $25,2 \%$ & $34,9 \%$ & $20,3 \%$ & $30,8 \%$ & $27,5 \%$ \\
\hline & \multirow{2}{*}{ Muy alta } & 20 & 9 & 6 & 6 & 41 \\
\hline & & $15,3 \%$ & $10,8 \%$ & $8,7 \%$ & $11,5 \%$ & $12,2 \%$ \\
\hline & \multirow{2}{*}{ Total } & 131 & 83 & 69 & 52 & 335 \\
\hline & & & $100,0 \%$ & $100,0 \%$ & $100,0 \%$ & $100,0 \%$ \\
\hline
\end{tabular}

Fuente: elaboración propia 
Para complementar el análisis, en la Tabla 4 se presentan los residuos estandarizados que se utilizan para mostrar las categorías que están relacionadas con más fuerza. Por lo tanto, los residuos más fuertes están en el tema áreas de preservación ambiental, que presenta un residuo positivo de 2.9 en baja visibilidad; legislación muestra residuo positivo de 1.9 en categoría de visibilidad media; y política de devastación, residuos de 2.9 positivos en la categoría de alta visibilidad. Esto demuestra que hay un predominio de estos temas en estas categorías y una tendencia a aparecer relacionados.

Los otros residuos no presentan valores por encima del límite crítico de +- 1.96. Esto demuestra que, a diferencia de la Gazeta do Povo, donde todos los temas tienden a aparecer equilibrados entre las categorías de visibilidad, en Folha de Sao Paulo el análisis de los residuos señala que algunos temas tienden a ganar más visibilidad que otros. Los textos acerca de las políticas contra la devastación tienen más visibilidad que los que hablan de la preservación del medioambiente, aunque que estos últimos aparezcan más veces.

Por lo tanto, en la FSP dominan textos menos visibles, como ya se señaló anteriormente, y algunos temas tienen relación positiva con esta categoría. Estos datos también ayudan a confirmar la hipótesis inicial, porque así como los temas no aparecen siempre de una manera equilibrada, algunos tienen más visibilidad que otros. Sin embargo el asunto que tiende a aparecer en textos de poca visibilidad es preservación, que estuvo en 170 textos (38,1\% de las entradas), pero, de estas, 75 (44,1\%) figuran en baja visibilidad. Es decir, los textos son fragmentados, pequeños y superficiales en su contenido. Muchas veces se trata de asuntos cotidianos que presentan solamente los hechos, sin una discusión posterior

Los otros temas, tales como legislación y política contra la devastación, que representan $11.2 \%$ y $14.3 \%$ de las entradas respectivamente, tienden a aparecer más en textos de visibilidad media y alta. Una explicación es que lo que más aparece cuando el periódico habla sobre devastación es la Amazonía y los problemas de deforestación. Aunque esté lejos de las personas, despierta más atención y es un problema que es monitoreado por el gobierno y que también hace parte del Pnma II. Entonces, cuando hay políticas para la Amazonía, ganan más espacio en la cobertura hecha por la prensa. Y el contenido que más ganó espacio cuando el periódico FSP habló acerca de legislación fue el Código Florestal; como estuvo en gran debate en la sociedad, también ganó más protagonismo en la prensa nacional. Por lo tanto, se puede concluir que temas que aparecieron más tendieron a tener menos visibilidad. Los que aparecieron pocas veces, cuando ganaron espacio en los medios de comunicación recibieron más visibilidad y una cobertura más amplia.

\section{2 COBERTURA SEGÚN LAS ETAPAS DEL CICLO POLIITICO}

Para complementar la investigación, también se analiza en qué parte del ciclo político las acciones del gobierno aparecen en los periódicos y tienen una mayor visibilidad. La Tabla 5 muestra las frecuencias de los datos y la cantidad de texto para cada etapa de la política pública. Tanto en la FSP como en Gazeta do Povo los datos presentan centralidad de la cobertura en dos momentos distintos: agendamiento y preparación, y ejecución.

\section{Tabla 5. Frecuencias de las etapas del ciclo político}

\begin{tabular}{|l|c|c|c|c|}
\hline \multirow{2}{*}{$\begin{array}{l}\text { Pasos del Ciclo de las Políticas } \\
\text { Públicas }\end{array}$} & \multicolumn{2}{|c|}{ Folha de São Paulo } & \multicolumn{2}{c|}{ Gazeta do Povo } \\
\hline Agenda y Preparación & Frecuencia & $\%$ & Frecuencia & \% \\
\hline Formulación & 159 & $\mathbf{3 5 , 7}$ & 104 \\
\hline Implementación & 71 & 15,9 & 65 & 17,7 \\
\hline Ejecución & 16 & 3,6 & 24 & 6,5 \\
\hline Acompañamiento & 130 & 29,1 & 98 & 26,7 \\
\hline Evaluación & 49 & 11,0 & 34 & 9,3 \\
\hline Indefinida & 13 & 2,9 & 21 & 5,7 \\
\hline Total & 8 & 1,8 & 21 & 5,7 \\
\hline
\end{tabular}

Fuente: elaboración propia 
En esta investigación se observó que los temas que más aparecen en cantidad de textos son los que tienen menos visibilidad; es necesario que los textos sean destacados para llamar la atención del lector. También encontramos que hay grandes diferencias entre los temas que están en un periódico y en otro: 'sostenibilidad' aparece más en la Gazeta do Povo y 'preservación de las áreas del medioambiente', en la FSP. Estos datos pueden ser explicados por la relación de la prensa con su entorno. 'Sostenibilidad' aparece más en la Gazeta porque está relacionada con la característica de la ciudad: 'preservación' aparece más en la FSP porque el periódico da importancia a los problemas medioambientales de las ciudades más grandes, como San Pablo.

Como podemos ver, el paso que más se destaca en la cobertura de ambos periódicos es 'agendamento y preparación', que tiene un papel más importante en el debate público, ya que precede las decisiones de los políticos Y lo que explica la presencia de este paso como el más citado, en términos de entrada de texto, es que casi siempre es muy polémico e implica la posición de la élite política en la materia, convirtiéndose en un tema relevante de los valores/noticia.

La explicación para la presencia del paso 'ejecución', que es el segundo que más aparece, es que se trata de acciones que están siendo ejecutadas, que es un trabajo más técnico. Demuestra también las actividades del gobierno, es decir, son textos que se ponen en la prensa porque son resultado de la labor de los periodistas que trabajan para el gobierno y tienen como función enviar pequenãs notas para la prensa, y estas son publicadas. Estos textos no contribuyen al debate público, porque no agregan una gran cantidad de información para los lectores y así no ayudan a formar su opinión o a discutir sobre el tema.

Los pasos 'acompañamiento y evaluación', que también tendrían impacto en el debate público, son los que menos aparecieron. En FSP están en el 11\% y 2.9\% de las entradas, respectivamente; y en la Gazeta do Povo los dos tienen un $5.7 \%$ de los textos. Es importante señalar que, aunque la literatura acerca de las polítcas públicas considera las etapas finales como esenciales para la definición de las nuevas acciones, estos dos pasos aparecieron muy poco en los dos periódicos. El debate acerca de la evaluación de las políticas es importante, porque muestra la eficacia o ineficacia de las acciones.

Después de encontrar que algunas etapas están más presentes en la cobertura de los dos medios, la Tabla 6 muestra la relación de las etapas del ciclo de la política con el índice de visibilidad. El objetivo es identificar si las etapas que aparecen más son las que ganan más visibilidad. La prueba de Chi-cuadrado encontró que la Gazeta do Povo no mostró una relación significativa, quedándose bajo el límite crítico de 0.05. El nível de significación resultante fue 0.327 , con un coeficiente de 13,604 . Por lo tanto, se concluye que los valores entre las categorías de variables se presentan de una manera equilibrada. En el caso de la FSP, la prueba de Chi-cuadrado encontró una significación de 0.000 , muy debajo del limite crítico. Esto nos permite identificar las relaciones más fuertes entre algunas categorías de las dos variables que aparecen en la tabla. El coeficiente fue de 47.840 .

Para el análisis, se le dará enfasis a los datos de la FSP, ya que la Gazeta do Povo no mostró relación entre las dos variables y sus categorías. Como se observa en la Tabla 6, la etapa de 'ejecución' de la política, la segunda que tuvo el mayor número de entradas, aparece con un $51,5 \%$ en la categoría de muy bajo. Es decir, a pesar de tener un gran número de textos, aparecen con muy poca visibilidad. Muchas veces los temas de esta categoria están en notas que apenas relatan los hechos diarios, por lo tanto no presentan discusión, fuentes de información ni imágenes. 
Tabla 6. Relación entre la visibilidade y las etapas del ciclo político

\begin{tabular}{|c|c|c|c|c|c|c|}
\hline & & $\begin{array}{c}\text { Agenda/ } \\
\text { Preparación }\end{array}$ & $\begin{array}{l}\text { Formulación / } \\
\text { implementación }\end{array}$ & Ejecución & $\begin{array}{c}\text { Acompañamiento/ } \\
\text { Evaluación }\end{array}$ & Total \\
\hline \multirow{17}{*}{$\begin{array}{l}\text { Folha de São } \\
\text { Paulo }\end{array}$} & \multirow{3}{*}{ Muy baja } & 44 & 19 & 67 & 8 & 138 \\
\hline & & $27,7 \%$ & $21,8 \%$ & $51,5 \%$ & $12,9 \%$ & $31,5 \%$ \\
\hline & &,- 9 & $-1,6$ & 4,1 & $-2,6$ & \\
\hline & \multirow{3}{*}{ Baja } & 44 & 20 & 16 & 13 & 93 \\
\hline & & $27,7 \%$ & $23,0 \%$ & $12,3 \%$ & $21,0 \%$ & $21,2 \%$ \\
\hline & & 1,8 & .4 & $-2,2$ &, 0 & \\
\hline & \multirow{3}{*}{ Promedio } & 19 & 15 & 11 & 11 & 56 \\
\hline & & $11,9 \%$ & $17,2 \%$ & $8,5 \%$ & $17,7 \%$ & $12,8 \%$ \\
\hline & & & 1,2 & $-1,4$ & 1,1 & \\
\hline & \multirow{3}{*}{ Alta } & 38 & 26 & 30 & 26 & 120 \\
\hline & & $23,9 \%$ & $29,9 \%$ & $23,1 \%$ & $41,9 \%$ & $27,4 \%$ \\
\hline & &,- 8 & .4 &,- 9 & 2,2 & \\
\hline & \multirow{3}{*}{ Muy alta } & 14 & 7 & 6 & 4 & 31 \\
\hline & & $8,8 \%$ & $8,0 \%$ & $4,6 \%$ & $6,5 \%$ & $7,1 \%$ \\
\hline & & .8 & .3 & $-1,1$ &,- 2 & \\
\hline & \multirow{2}{*}{ Total } & 159 & 87 & 130 & 62 & 438 \\
\hline & & $100,0 \%$ & $100,0 \%$ & $100,0 \%$ & $100,0 \%$ & $100,0 \%$ \\
\hline \multirow{12}{*}{$\begin{array}{l}\text { Gazeta do } \\
\text { Povo }\end{array}$} & \multirow{2}{*}{ Muy baja } & 21 & 22 & 24 & 13 & 80 \\
\hline & & $20,2 \%$ & $24,7 \%$ & $24,5 \%$ & $23,6 \%$ & $23,1 \%$ \\
\hline & \multirow{2}{*}{ Baja } & 21 & 15 & 22 & 9 & 67 \\
\hline & & $20,2 \%$ & $16,9 \%$ & $22,4 \%$ & $16,4 \%$ & $19,4 \%$ \\
\hline & \multirow{2}{*}{ Promedio } & 20 & 17 & 16 & 9 & 62 \\
\hline & & $19,2 \%$ & $19,1 \%$ & $16,3 \%$ & $16,4 \%$ & $17,9 \%$ \\
\hline & \multirow{2}{*}{ Alta } & 26 & 22 & 33 & 14 & 95 \\
\hline & & $25,0 \%$ & $24,7 \%$ & $33,7 \%$ & $25,5 \%$ & $27,5 \%$ \\
\hline & \multirow{2}{*}{ Muy alta } & 16 & 13 & 3 & 10 & 42 \\
\hline & & $15,4 \%$ & $14,6 \%$ & $3,1 \%$ & $18,2 \%$ & $12,1 \%$ \\
\hline & \multirow[t]{2}{*}{ Total } & 104 & 89 & 98 & 55 & 346 \\
\hline & & $100,0 \%$ & $100,0 \%$ & $100,0 \%$ & $100,0 \%$ & $100,0 \%$ \\
\hline
\end{tabular}

Fuente: elaboración propia 
Lo contrario ocurre con el paso 'acompañamiento y evaluación' de políticas, que apareció muy poco, pero el 41,9\% se concentró en la categoría de alta visibilidad. Esto puede explicarse por el hecho de que estos contenidos implican nuevos y mejores debates entre los actores sociales. Por lo tanto, los textos no son tan fragmentados y ganan más protagonismo. Se observa aún que hay residuos negativos en la relación entre este tema y la categoría muy baja visibilidad, es decir, no tiende a aparecer relacionado con aquella categoría. La visibilidad de este paso es muy diferente a la de la etapa de 'ejecución'.

Las etapas 'agendamineto y preparación' y 'formulación' parecen más divididas entre las cinco categorías. Sin embargo, se subraya que la agenda y la preparación tienen su mayor porcentaje en bajo y muy bajo, ambos con $27,7 \%$ de los textos. La 'formulación' y la 'implementación' tienen su mayor porcentaje, 29,9\% en visibilidad alta. Son textos que casi siempre hablan de la legislación y los proyectos que han sido aceptados por el gobierno. Sin embargo, son los pasos 'ejecución' y 'acompanãmiento y evaluación' los que tienen una mayor concentración de entradas en solo una categoría de la variable visibilidad y que presentan relaciones diferentes entre ellos.

Los datos muestran que, en general, son estas dos categorías -la 'ejecución' y el 'acompañamiento y evaluación'las que representan una asociación significativa entre las variables y hay una tendencia a que los textos de la 'ejecución', a pesar de la gran cantidad, sean menos visibles en la página del periódico, mientras que los textos del 'acompañamiento y evaluación' tienen mayor visibilidad.

En esta investigación se observó que los temas que más aparecen en cantidad de textos son los que tienen menos visibilidad y lo contrario ocurre con los que aparecen pocas veces. A pesar de que la redundancia es una característica del proceso de 'agendamiento', es necesario que los textos sean destacados para llamar la atención del lector. 'Agendamiento y formulación' no tiene relaciones significativas con las categorías de índice de visibilidad, pero la etapa de 'agendamiento' recibe muchas entradas y tiende a aparecer más prominente que 'ejecución'. Es decir, aunque los textos técnicos aparezcan, tienden a tener poco espacio y solamente mostrar los hechos, sin ponerlos en debate.

\section{CONSIDERACIONES FINALES}

En este trabajo se ha identificado la forma que los periódicos de Brasil -Gazeta do Povo y FSP- hacen la cobertura de las políticas públicas ambientales durante la implementación y ejecución del Pnma II, durante 10 años. Los primeros datos mostraron diferencias en el número de entradas: en FSP hay más entradas que en la Gazeta do Povo, pero en esta última los textos tienden a tener más espacio, es decir, el tema puede ser mejor sistematizado cuando hay más espacio para la confrontación de ideas, uso de fuentes y de imágenes, lo que significa que los lectores de este diario reciben más información. Además del tamaño, comparando las categorías del índice de visibilidad de los dos periódicos, se observa que en la FSP los textos tienen más visibilidad en comparación con la Gazeta do Povo. Estos datos, como ya fue discutido, tienen explicaciones de dos tipos: externa a los periódicos e institucional. Estos niveles de influecia están en la literatura y pueden ser usados para la búsqueda de posibles explicaciones para los datos de la investigación.

La FSP, como es un periódico de ciculación nacional, tiene un grupo de temas mayor que la Gazeta para definir su pauta. Y cuando los periodistas hacen la selección, hay otros temas que ganan más protagonismo en las páginas. Es diferente en los periódicos màs pequeños, donde hay pocos temas y así las políticas tienden a tener más espacio. Además, Curitiba, donde la Gazeta es producida, es conocida como la capital ecológica, por lo tanto algunos temas tienden a aparecer porque hay acciones del gobierno en Curitiba relacionadas con la caracteristica de la ciudad y así las politicas ganan más importancia. Y Paraná, el estado donde el periódico Gazeta es producido, también tiene muchos problemas ambientales, ya que tiene grandes zonas agrícolas y gran parte de los bosques fueron deforestados, lo que siempre genera más discusión entre las personas que viven en estos ambientes.

También encontramos que hay grandes diferencias entre los temas que están en un periódico y en otro. Se observó que dos de ellos tienen un mayor número de entradas: 'sostenibilidad' y 'preservación de las áreas del medioambiente', siendo que el primero aparece más en la Gazeta do Povo y el segundo, en la FSP. Esta información muestra que no hay equilibrio en la cobertura de los temas en los periódicos analizados, lo que confirma la hipótesis inicial del trabajo: que algunos temas tienen más espacio que otros en la cobertura. Pero estos datos también pueden ser explicados por la relación de la prensa con el ambiente. 'Sostenibilidad' aparece más en la Gazeta porque está relacionada con la caracteristica de la ciudad, y 'preservación' aparece más en la FSP porque el periódico escribe más sobre los problemas medioambientales de las ciudades más grandes, como San Pablo. 
Además, se observó que en la FSP el tema áreas de preservación ambiental tiende a aparecer muchas veces, pero en textos de poca visibilidad y que las políticas contra la devastación tienen más posibilidades de aparecer en textos de alta visibilidad. Así, en la FSP hay una inversión, porque el tema que más aparecía fue aquel que tuvo poca visibilidad, mientras aquel que aparecía pocas veces tenía más visibilidad. 'Preservación' aparece muchas veces, pero siempre en pequeñas notas que sólo proporcionan informaciones cortas. Así, no hay mucha discusión en los textos, que son informes breves de naturaleza episódica. Ya en los textos acerca de devastación hay un debate más amplio sobre los resultados de las acciones, con la presencia de más fuentes. Esto significa que aparecer muchas veces en el periódico no significa mayor visibilidad, como se ha demostrado en este estudio de caso. Esto no ocurrió con la Gazeta do Povo, donde los temas estaban mejor distribuidos.

Acerca de la presencia de las etapas de las políticas, ocurrió la misma cosa. Los datos muestran la centralidad de la cobertura en dos momentos distintos: 'agendamiento' y 'ejecución'. Agendamiento se considera el momento en que los medios de comunicación tienen un papel más eficaz en el proceso político, mientras que en la 'ejecución' su papel es secundario, ya que sólo describe las medidas adoptadas por el gobierno. Los primeros datos muestran que los periódicos cumplen su rol de discutir temas sobre políticas públicas. Se observó también que en la FSP el etapa de la 'ejecución' esta centrada con 51,5\% de las entradas de visibilidad muy baja y 'acompañamiento y evaluación de las políticas' -uno de los que menos aparecióconcentra $41.9 \%$ en la categoría alta visibilidad.
En general, el texto muestra que las políticas tuvieron más protagonismo y visibilidad en la Gazeta do Povo, a pesar de publicar menos noticias del tema. Esto significa que en este periódico es más probable que el tema reciba la atención del lector y esté presente en la agenda pública. Pero se señala que, en los dos periódicos, tanto las etapas como los temas no aparecen todos iguales, lo que confirma la hipótesis inicial y puede ser explicado porque algunas estapas tienen más valores/noticias, es decir, llaman más la atención de los periodistas y algunas también llegan en la prensa porque hacen parte de la esfera política. Como señalan algunos autores, hay proximidad entre la esfera política y mediática. Por lo tanto, no todos los temas pueden, mediante el agendamiento, hacer parte de la agenda pública, ya que algunos están prácticamente ausentes de la prensa, aunque sean importantes para la sociedad.

En el análisis fue posible idenficar y elaborar algunas hipótesis de respuestas para las difirencias entre los temas en los periódicos: están relacionadas con las condiciones externas que influyen en la produción y también con las caracteristicas de los periódicos y periodistas. De hecho, los aportes que Shoemaker y Vos (2009) hicieron sobre los niveles de influencia de los factores internos y externos en la redacción pueden explicar los resultados encontrados en esta investigación. Es decir, el trabajo empírico utiliza la bibliografía para su base teórica - para justificar el análisispero también la usa para producir las interpretaciones de los resultados. Y como se puede percibir, las explicaciones son más institucionales y consideran el ambiente de produción de las noticias.

\section{SOBRE LOS AUTORES:}

Emerson Urizzi Cervi. Doctor en Ciencia Política, Instituto Universitário de Pesquisas do Rio de Janeiro. / Profesor investigador del Departamento de Comunicación y Periodismo, Universidad Estadual de Ponta Grossa y del Departamento de Ciencias Sociales, Universidade Federal do Paraná. / Coordinador del grupo de investigación en Medios y Política, UEPG.

Michele Goulart Massuchin. Mestranda en Ciencias Políticas, Universidade Federal do Paraná (UFPR). Miembro del grupo de investigación en Comunicación política y Opinión pública, UFPR. 
Abreu, M. S. (2006). Quando a palavra sustenta a farsa: o discurso jornalístico do desenvolvimento sustentável. Florianópolis: UFSC.

Aguiar, L. A. (2005). Representações da crise do meio ambiente no jornalismo científico. Paper presentado en XVIII Intercom Nacional, Brasil.

Alves, J.M. (2001). O papel da mídia na informação Ambiental. Disponible en: www.unitau.br/scripts/prppg/ humanas/.../opapelmidia-N2-2001.pdf

Arruguete, N. (2005). Los medios de comunicación y la formación de la agenda pública. Revista de comunicaión Unisinos. 41, XIX.

Bauer, M. (2002). Análise de conteúdo clássica: uma revisão. En Bauer, M. W. y Gaskell, G. (org.). Pesquisa qualitativa com texto, imagem e som: um manual prático. Petrópolis: Vozes.

Bonfiglioli, C. P. (2006). Discurso, imagem e cultura: a representação do protocolo de Kyoto. Estudos em Jornalismo e Mídia, UFSC.

Bueno, W. C. (2004). Jornalismo Ambiental: navegando por um conceito e por uma prática. Disponible en: www. ecoviagem.com.br/fique.../jornalismo-ambientalnavegando-por-um-conceito-e-por-uma-pratica.

Capella, A. (2005). Formação da Agenda Governamental: Perspectivas Teóricas. Trabajo presentado en XXIX Encontro Anual de ANPOCS.

Cervi, E. (2009). Métodos quantitativos nas ciências sociais: uma abordagem alternativa ao fetichismo dos números e ao debate com qualitativas. En Ayres, J. B. (Org.) Pesquisa Social: reflexões teóricas e metodológicas. Ponta Grossa: Toda Palavra.

Cervi, E. (2010). Opinião Pública e comportamento político. Curitiba: Ibpex
Davis, J. A. (1976). Levantamento de Dados em Sociologia: uma análise estatística elementar. Rio de Janeiro: Zahar Editores.

Habermas, J. (2006) Political Communication in mídia Society: Does Democracy still enjoy na epistemic dimension? The impact of Normative Theory on Empirical Research. Revista Communication Teori 16, 411426

Lima, E. S. (2002). A importância da mídia na consciência ambiental. Disponible en: http://www. jornalismocientifico.com.br.

Lima, L. (2008). As Políticas Públicas para o Meio Ambiente. Recanto das Letras.

Maia, R. (2002). Mídia e deliberação pública: mediações possíveis. Texto presentado en GT Comunicação e Política, XII Reunión anual da Compós.

McCombs, M. (2009). A Teoria da agenda: a mídia e a opinião pública. Editora: Vozes.

McCombs, M; Shaw, D. (1972). The Agenda-setting Function of Mass Media. Public Opinion Quarterly, 36, 176-87.

Miguel, L. F. (2002). Os meios de comunicação e a prática política. Revista de Cultura e Política, 55/56.

Mirrales, A. M. (2004). Periodismo, opinión pública y agenda ciudadana. Bogotá: Norma.

Shoemaker, P J; Vos, T. (2009). Gatekeeping Theory. New York: Routledge.

Souza, J. (2005). Comunicação, meio ambiente e práticas culturais: um estudo sobre o alto Uruguai catarinense. Disponible en: http://biblioteca.universia.net/ficha. do? id=38067114

Trigueiro, A. (2005). Mundo Sustentável. São Paulo: Globo. 
Trigueiro, A. (2008). Meio ambiente na idade mídia. Campinas: Armazém do Ipê.

Tautz, C. (2004). Oxigênio para a energia: Entenda a Idéio de um "Jornalismo para o desenvolvimento".En Vilas Boas, S. (Org.). Formação \& Informação Ambiental: jornalismo para iniciados e leigos. São Paulo: Summus.

Urban, T. (2006). Um novo olhar da mídia sobre o meio ambiente. Disponible en: http://www.comscientianimad. ufpr.br/2006/01/pontodevista/tereza_urban_u m_novo_ olhar.pdf.

Villar, R. (1997). Jornalismo Ambiental - Evolução e Perspectivas. Disponible en: www.agirazul.com.br/artigos/ jorental.htm. Acesso em: 20 de setembro de 2008.
Villar, R. (2004). Cidades em mutação: menos catástrofes e mais ecojornalismo. En Vilas Boas, S. (Org.). Formação E Informação Ambiental: jornalismo para iniciados e leigos. São Paulo: Summus.

Tosi, J y Villar, R. (2001). Jornalismo Ambinetal: onde estão as faculdades de comunicação? Disponible en: www. portoalegre.rs.gov.br/ecos/revistas/ecos19/opiframe.htm.

Wolf, M. (2001) Teorias da Comunicação: Mass Media: contextos e paradigmas, novas tendências, efeitos a longo prazo, o newsmaking. Lisboa: Presença, 2001. 